\title{
Exploring Reactions to Widespread Energy Monitoring
}

\author{
James A. Colley ${ }^{1}$, Benjamin Bedwell ${ }^{2}$, Andy Crabtree ${ }^{1}$, and Tom Rodden ${ }^{1}$ \\ ${ }^{1}$ Mixed Reality Lab, School of Computer Science, \\ University of Nottingham, Nottingham, UK, NG8 1BB \\ ${ }^{2}$ Horizon Digital Economy Research \\ University of Nottingham, Nottingham, NG7 2TU \\ \{psxjac, first.last\} @nottingham.ac.uk
}

\begin{abstract}
This paper explores the measurement, apportionment and representation of widespread energy monitoring. We explicate the accountability to users of the data collected by this type of monitoring when it is presented to them as a single daylong picture. We developed a technology probe that combines energy measurement from the home, workplace and the journeys that connect these spaces. Through deployment of this probe with five users for one month we find that measurement need not be seamless for it to be accountable; that apportionment is key to making consumption for communal spaces accountable and that people can readily make useful inferences about their energy consumption from daylong pictures formed from widespread monitoring. Finally, we present four issues raised by the probe - the nature of real world monitoring, the dynamic and social nature of apportionment, disclosure of energy data and alignment of incentives with consumption - that need to be addressed in future research.
\end{abstract}

Keywords: Distributed energy monitoring, measurement, apportionment, representation, technology probe.

\section{Introduction}

Energy security and sustainability are established as global societal challenges and have prompted a variety of responses and initiatives. Technical and operational responses have turned to digital technologies, to capture the consumption of energy and to make this available to stakeholders to optimise energy use. The ability of the technology that captures and makes available information on energy use has been a major focus for researchers in HCI and ubiquitous computing. Researchers have sought to promote awareness of energy consumption as a way of encouraging changes in peoples' behaviour [7]. A critical feature of much of this work has been the development and deployment of devices and interfaces that surface energy consumption in order to make consumption accountable to consumers and thus motivate them to reduce energy use. Examples include technologies for the home [13, 14], transport [10] and the workplace [23].

The framing of the energy challenge within HCI in terms of surfacing consumption in particular settings has not passed without criticism. Pierce and Paulos [22], for 
example, have called for a shift beyond residential energy feedback systems, highlighting a disconnect between current HCI energy research with its emphasis on in home display; and energy research that adopts a much broader perspective. Others have questioned the emphasis on measurement and persuasion as a narrowing of the concerns involved in sustainability [2]. However, considerable efforts persist in making energy measurement even more pervasive and fine-grained. For example, research is emerging within ubiquitous computing that focuses on capturing a rich picture of energy use across a broad range of settings [e.g, 10,21]. As these technologies for energy data capture mature, a number of researchers have offered the vision of a personal energy monitor that captures peoples' energy footprint throughout the day [16].

We are interested in understanding how people may react to a future world where information about their energy use throughout the day, across the multiple spaces they operate in, is captured and surfaced. What does it mean to surface energy consumption and make it accountable across and between the different places that people inhabit and how will people react to this? By way of an initial answer, this paper presents the development and study of a technology probe focusing on the measurement and surfacing of energy consumption across and between multiple spaces.

The probe combines a mobile device carried by users that works in tandem with energy monitoring systems in different spaces. The probe monitors consumption in users' homes and workspaces, and records the time spent in these places along with journeys made by users. Three key research issues drove the design of the probe:

1. Measurement. How widespread and how fine-grained does measurement need to be to surface energy consumption across and between multiple spaces?

2. Apportionment. How should measured energy consumption be apportioned across and between multiple spaces?

3. Representation. Will representations of apportionment make energy consumption across and between spaces accountable to consumers, and in what ways?

The probe was deployed in the wild with five participants over a period of one month. At the end of the month users were presented with a web-based representation of their data displaying consumption across their home and work lives as well as the journeys that are taken. The data was used to drive semi-structured interviews with each participant in order to understand the representation and its potential affordance to human reasoning about energy consumption.

The results from our probe suggest that while it is not possible to measure all of a person's daily consumption, partial measurement alongside user inference is sufficient to make the consumption accountable to users. However, that ability to make energy information available across different contexts raises a number of significant tensions that may cause widespread measurement and individual accountability to act as a significant source of demotivation for some. This is particularly true when we consider the differences between the roles of a user in domestic and workplace energy consumption. 


\section{Existing Approaches towards Surfacing Consumption}

Recent research in HCI has focused on surfacing energy consumption to encourage consumers to change their behaviour. The basic premise is that surfacing energy use makes consumption visible and accountable to consumers and, in turn, enables them to take appropriate action. To date, there are two key approaches towards surfacing consumption: space-centric approaches and person-centric approaches.

\subsection{Surfacing Energy Consumption within Spaces}

A common approach has been to surface energy consumption within a space. This often relies on measuring the consumption of appliances, and results in the creation of artefacts or persuasive displays that surface appliance-based energy consumption data and imply accountability. Thus far, these feedback systems have focused mainly on domestic spaces. Examples include the power aware cord [13], the power socket [17], the watt bot [19], light bulb radiators [14] and the minimalist in-home energy consumption display [24]. While novelty effects have been seen, significant long-term changes in behaviour have not yet been demonstrated by these HCI-led initiatives. However, long term studies of standard in home displays (IHDs) have demonstrated an impact. For example, in a review of 36 studies of feedback on behaviour between 1995 and 2010, Ehrhardt-Martinez et al. found an average of 9.2\% reduction in electricity usage as a result of the use of an IHD [8].

Work within ubiquitous computing has focused on low-cost sensing technologies to capture more detailed information about domestic consumption. Patel et al. [21] have developed a system that makes use of electrical noise signatures on residential power circuits to detect the switching of particular appliances. This demonstrates how very specific high-resolution sensing can allow micro-level events in the home to be surfaced. Abrahamse et al. [1] reviewed thirty-eight studies around technologies to capture and present energy consumption. It is notable that none surfaced consumption from more than one space and that the predominant focus was on surfacing energy use in the home. An additional review of studies of 'eco-feedback' by Froehlich et al. [10] echoes the concern that studies of such technologies seldom consider the connected nature of consumption in everyday life and that people are therefore made accountable for consumption in their homes in isolation from other spaces. As the majority of consumption still occurs outside the home ${ }^{1}$, we are interested in exploring how measurement and feedback technologies might include, but also go beyond domestic infrastructures to consider energy use across multiple spaces.

\subsection{Surfacing Personal Energy Consumption}

Efforts to surface consumption within a space have been complemented by other efforts to surface personal consumption. The UbiGreen application [10] makes use of mobile phones connected to Mobile Sensing Platform devices to semi-automatically

\footnotetext{
${ }^{1}$ E.g., $71 \%$ of energy is consumed outside of the home in the UK [6].
} 
infer consumers' transport modes, exploiting various visualisations to then make carbon consumption accountable to the user to promote greener travel choices. Related work by Mun et al. makes use of map matching and hidden Markov models to build a "Personal Environmental Impact Report", which visualizes the carbon intensity of trips. This work is technically impressive in the way that it combines multiple data sources however there is no data on its users' reactions [20]. Surfacing personal energy use has also become a key feature in government initiatives. These have promoted concepts such as the carbon footprint and Personal Carbon Trading Schemes [e.g., 9]. The underpinning idea is that individuals are made accountable for their carbon footprint by having to deduct their personal portion of consumption away from an allocated quota based on an overall national cap.

An alternative person-oriented approach seeks to understand the human practices that shape energy consumption and sustainable behaviour [5]. This has highlighted the broad character of personal energy consumption.

"Traditionally, we have taken a within household approach to infrastructure, emphasizing systems that will make our homes smart. But households are connected to a variety of infrastructures beyond the home ... householders desire insight into ... resource usage and more visible real-time information on within the home resource consumption. They also desire more information on between-homes consumption" (ibid.)

Chetty and Grinter's study draws attention to the distributed nature of energy consumption and the concomitant implication that personal energy consumption needs to bridge the home and other spaces that people inhabit if it is to be of broad utility. This view is supported by research that has explored the development of the "personal energy meter' [16]. This trades on a vision of a widespread energy-monitoring infrastructure to provide people with a personal account of energy use across their lives.

\section{The Technology Probe}

Existing approaches to surfacing energy consumption and making it accountable to users suggest the need to move beyond isolated contexts to surface energy use across multiple spaces. While there is a broad turn towards capturing detailed information about energy consumption in more locations through the widespread introduction of smart meters, the distributed character of energy consumption raises a host of challenges. How might we surface consumption across the multiple spaces we inhabit? How might we make consumption at work accountable to users? How might we understand energy use in public spaces that are seldom monitored?

Capturing information is one thing, making it into a useful and usable resource for action another. Making use of existing monitoring infrastructures, the aim of this paper is to contribute to an increased understanding of how people might respond when energy consumption from multiple spaces is surfaced and presented to them. We address the challenge through the development and deployment of a technology probe. Technology probes are an extension of the cultural probe methodology [12], which seeks to "provoke inspirational responses" from potential users as a resource 
for design. Technology probes on the other hand are "thought-provoking technologies" - provoking in the sense that they enable people to reason about how future visions "do or don't fit into their lives" [18]. They also have the added benefit that they enable data logging. While involving design and implementation, technology probes are not intended to be prototype solutions amenable to usability testing.

"They are not changed during the use period based on user feedback. In fact, a deliberate lack of certain functionality might be chosen in an effort to provoke the users" (ibid.)

Our interest is not in whether the technical intervention is usable, but rather in the principles underpinning future widespread monitoring technologies. The probe seeks to elicit views on capture and attribution and how we might design choices of energy apportionment. This is reflected in a number of explicit design choices. Most notably we have chosen a simplistic means of apportionment to provoke users to comment on their own personal accountability for energy use. We do not seek to record and represent the fact that different inhabitants will consume energy in a space in different ways. Likewise, we do not consider that individuals may stake claim to energy consumed in spaces that they do not inhabit (e.g., a dishwasher timed to commence while its owner is at work). We are interested in the strategies of apportionment that make sense to users and how these design choices might be reasoned about. Thus, rather than build a variety of apportionment models into the probe, we used a deliberately crude but consistent approach as starting point, which we could then open up to discussion during interview.

The probe itself combines three key elements designed through a series of iterative refinements: multi-site energy measurement, energy apportionment, and representation of personal energy consumption.

We describe each of these in turn below, before moving on to consider the results of deployment and users' reactions to the probe.

\subsection{Measurement}

Monitoring centred on a cloud-based logging service that received data from three distinct types of data source: home energy consumption collected via a deployed meter; workplace energy consumption collected by interfacing to a building management system, and the reported points of journeys taken, collected using a smartphone.

\section{Monitoring the Home}

The home monitoring kit consisting of a current transformer monitoring clamp and a plug computer was used to record the rate of consumption in participant's homes. Data was sent to a secure cloud-based server every 6 seconds. On the server side, power readings were aggregated once every hour down to a resolution of 1 mean reading for every 5 minutes to reduce the computational expense of querying the data. 


\section{Monitoring the Workspace}

Energy consumption in the various workplace buildings in our study was logged by an estate management department using an existing building management system (BMS). Automated BMS export functions were used to deliver data to the probe's cloud-based logger. The BMS monitors are coarse at a temporal level, as they are designed to generate data for high-level monthly reports, providing readings per building every 30 minutes. Furthermore, each building's readings vary in resolution: for some, lighting and socket power readings per floor are recorded and for others only the consumption of the whole building is recorded. Newer buildings tend to have a higher resolution of monitoring compared to older ones, where retrofitting more monitors is deemed not cost-effective.

\section{Monitoring the Journey}

Location information is collected through a dedicated mobile phone application, which reports the users' location based on Wi-Fi positioning via Google's location service on the Android platform. Wi-Fi was chosen both because it provides a level of accuracy suitable to determine which building a user currently inhabits (between 6 and 20 meters on average) and because it works inside and outside of buildings, while not draining the battery significantly. The service typically provides location updates (consisting of latitude-longitude coordinates) every 30 seconds. The location is sent securely along with a unique identifier for the user to the cloud-based logger.

\subsection{Apportionment}

The apportionment process collates the data relevant to a particular individual for a particular day based on a set of monitored spaces. Monitored spaces are defined using 'geo-fencing', i.e. the definition and storing of polygons that represent the boundaries of workplace buildings and homes inside which energy consumption is being monitored. These polygons are cross-referenced with users' locations as they are reported by the logging application on their mobile phones to determine whether an individual is present in any of the monitored areas. If entry into an area is detected (i.e., the user's mobile device automatically 'checks-in') this event is logged; no further latitude-longitude coordinates are logged until the user's mobile leaves ('checks-out' of) the area.

This record of automated check-ins and check-outs provides a simple means of apportioning energy consumption data across multiple spaces. It is based on the crude assumption that every individual inhabitant of a monitored space is responsible for an equal proportion of the energy used in that space while they are present. As all inhabitants of a space were not monitored, individuals were made accountable for personal energy consumption equal to the total energy consumption of the space divided by the maximum occupancy of that space. This is obviously a crude presumption - it will allow high consumers to "free-ride" - and as such we would not necessarily propose it for a fully deployed system.

Discovering an appropriate model of apportionment has already gathered some significant research interest. For example, Hay et al. have investigated different 
models of apportionment for the workplace using access logs and energy monitoring to simulate policies for example individuals [15]. Our research interest focuses on the issues of intelligibility and accountability of apportionment. As such, a crude model that is simple to understand but potentially unfair allows us to takes a provocative stance to encourage reaction from users.

\subsection{Representation}

The way that data was represented to our participants was key in provoking them into thinking about the issues implicated in widespread energy monitoring, particularly the accountability of energy consumption when measured and apportioned across multiple spaces. We represented the data gathered during the deployment of the probe via a web-based 'personal energy dashboard', which was made available to participants during the interviews to surface and replay their energy usage and encourage them to reflect on their consumption patterns.

\section{The Personal Energy Dashboard}

The personal energy dashboard (Figure 1) consists of 4 components:

1. A map (Figure 1, Section 1) showing the trail that the user has left when they are in unmonitored space, the spatial boundaries of monitored spaces they have occupied, and consumption in the places they have occupied. The consumption is represented on the map by bars situated at the centre of the associated building with a height proportional to the energy consumption of the building for the time the user has occupied it. This map assists users in thinking about how they connect consumption infrastructures to their lives.

A day long bar chart (Figure 1, Section 2) with time on the horizontal axis and consumption on the vertical axis. This shows how consumption has varied as the user moved through different buildings throughout the day. No bar is shown if the

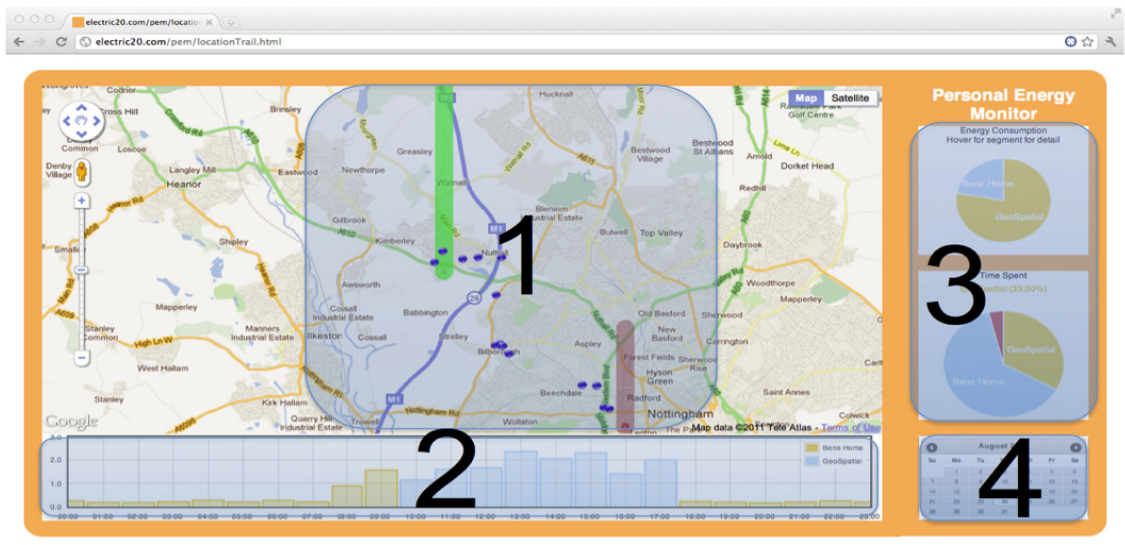

Fig. 1. The personal energy dashboard 
user has been in unmonitored space for an hour slot. The purpose of this chart is to show how consumption changes throughout the day depending on the spaces inhabited. It also demonstrates the apportionment done by the system so as to allow users to reflect upon and challenge it.

2. Two proportional pie charts (Figure 1, Section 3). One shows the proportion of consumption for the time spent in monitored space (Section 3, top). The other shows the segments of time spent in monitored buildings; this also includes a segment for time spent in unmonitored space (Section 3, bottom). This feature is intended to provoke views around apportionment and consumption across multiple infrastructures.

3. A date selector (Section 4, Figure 1), which allows the user to revisit previous days by navigating through the $\log$ on a day-by-day basis.

The display is intended as a focal point for our semi-structured interviews rather than as an interface to promote energy reduction. The aim is thus reflection on the underlying approach and systems rather than actionable energy reduction suggestions. The personal energy dashboard allows users to both reflect upon the time they spend within different monitored and unmonitored spaces throughout their day, and to view the consumption data that pertains to them within those spaces.

\section{The Probe Study}

The probe deployment involved five participants recruited via email within one workplace that was chosen because researchers could readily access data from the workplace's BMS. We installed monitoring equipment in each participant's home and provided a mobile phone that reported his or her location to our system. The participants included:

- George, a 27 year-old professional who is recently married. He often spends weeks living alone as his wife travels internationally on business. He works in an office from 9 to 5 most days and occasionally from home.

- Alice, a 31 year-old, married, middle-class office worker. She shares use of a car with her husband, but enjoys cycling to work when her husband needs the car. Alice works long hours in the office, but her work can occasionally spill into her home life.

- Cecile, a 34 year-old single woman from Europe. She generally works a 9-to-5 day in the office and has an active but distinctly habitual social life. She commutes by bicycle and is proud to consider herself both energy conscious and a low consumer of energy.

- Fred, a 32 year-old recently married professional who likes to keep his home and work life separate. He made the choice to live on the outskirts of the city where he works. He often drives to and from work but occasionally cycles when the weather is agreeable.

- Bill, 26 years old and currently in the final stages of his formal education. He commutes 150 miles (twice a week) by either car or train between his place of 
study and his girlfriend's hometown, where he spends the weekend and works a part time job for 1 day a week. ${ }^{2}$

The probe deployment lasted one month, during which time a rich set of data about participants' energy consumption and their location was logged. Data from the probe was presented to users through the personal energy dashboard, allowing them to browse through their daily energy consumption over the period of the deployment. Semi-structured interviews were used to understand the accountable character of monitoring and apportionment across and between multiple spaces or, to put it another way, to explore how amenable this kind of information is to human reasoning.

\section{Results}

Energy data was collected from all 5 participants' homes and 4 separate workplace buildings used by participants. Cumulatively, 183000 data points for energy consumption were recorded across all monitored spaces, and 4500 location reports outside of monitored space were recorded. This level of measurement allowed us to create energy dashboards that represented a month's worth of data for each participant.

The first and most obvious thing we noticed is that our participants spent a considerable proportion of their time in unmonitored space (11\%). This included commutes to and from work, time out socializing, visits to the country and holidays. Their remaining time was split between personal space $(62 \%)$ and the work place $(27 \%)$. There were obviously individual differences in this data reflecting different lifestyles. For example, Fred who lives furthest from his place of work had a larger proportion of time in unmonitored space, reflecting his daily commute. Similarly, Cecile had a large proportion of unmonitored space reflecting her regular attendance at social events. There were also significant differences between the working week and weekends, where our participants often spent $50 \%$ of their time in unmonitored spaces.

While our participants spent the majority of their time in personal space, most energy consumption actually took place in communal spaces. Over $63 \%$ was consumed in communal spaces where our individuals had limited control of their consumption, with less than $37 \%$ being consumed in personal space where they had direct control of consumption (see Figure 2).

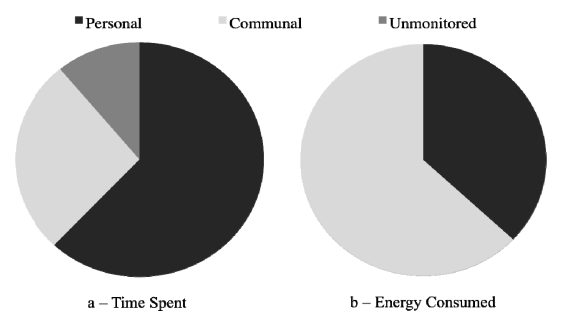

Fig. 2. Proportion of time (a) and energy consumed (b) in different spaces

\footnotetext{
${ }^{2}$ These are, of course, not the participants' real names.
} 
The collected data provoked responses from our participants to the measurement, apportionment and representation of energy data and these three themes provided the focus for our interviews.

\subsection{Measurement}

The probe was designed to explore how widespread and how fine-grained measurement needs to be to surface energy consumption across and between multiple spaces. Deployment of the probe suggests that collecting sensor data in the wild, particularly from multiple sources, results in 'seams' [3] appearing within the data. These are a result of the environment (e.g., when users move outside of and between monitored spaces) and unexpected events (e.g., when users let their phones run out of battery or leave their phone at work overnight). When this seamful data was presented to participants through the dashboard interface they responded with discussions around what the seams meant to them and how they affected the legibility of the data.

Alice: I can see there's no energy data here, but I can see where I was travelling to and from ... I did lots of travelling this day but by different means. I took the train to Liverpool then drove back. I can see that from the routes.

Rather than being seen and treated as unfathomable gaps in the data, participants were instead able to see their behaviour in the seams and infer usage. Discussions around the location trails that were recorded outside of monitored space revealed that participants were particularly good at recalling their travel habits and filling in the gaps when they existed. Sometimes this was done at a level of detail that would be difficult to achieve by sensing and measurement alone:

Fred: I took the train from there to there, then I cycled down [road $\mathrm{x}$ ].

Bill: That day I had to go a different way because of road works, it took me longer.

Participants demonstrated an understanding around issues of connectivity, how this impacted their data, and accepted that this is part of life.

George: It didn't really get when I was out in the countryside, but there's no consumption there anyway. It's all fields and cows.

We designed the probe to allow participants to collect data from each other's houses when they visited them. Figure 1, Section 3 shows the spaces in which consumption occurred; when participants visited each other's houses they were able to see the visited house's consumption as part of their own chart. Our intent here was to surface and explore the issues of leakage that might possibly occur with monitoring of this form and to raise issues of privacy with the users.

On measurement, we conclude that it is meaningful for participants to have consumption in the home and work place measured, a load reading every hour taken from these spaces allowed them to compare and contrast the varying consumption between the spaces that they passed through and reason about their accountability for consumption in these spaces. We also note that gaps in data are acceptable as long as seams are meaningful enough to be repaired by user inference. 


\section{$5.2 \quad$ Apportionment}

The probe was also designed to enable us to address the key question of apportionment and how it should be calculated across and between multiple spaces. Although we adopted a crude means of calculation, participants nevertheless expressed concerns over the level of control available over consumption in the home versus the workplace.

Alice: I can't turn off my computer at work, because I need it to work. I can't turn the heating down even though its too warm here, at home I can just turn the heating off!

Participants were able to read from the dashboard that the energy monitored for their time at work far outweighed the energy consumption for their time at home.

Bill: The work consumption makes my home look like a piss in the ocean.

However, people also elaborate rationales for these high loads in communal buildings, examples of which include recognizing that the energy consumption was necessary for health or a sense of shared responsibility for the provision of services to multiple users of buildings.

Fred: I think I do feel some responsibility for all the servers on at work all the time but the servers at work mostly serve my building so they're related to our work.

When discussing apportionment of energy with participants, a desire for a more detailed breakdown of energy consumption in communal spaces was sought to assist in their reasoning about the high levels of consumption.

George: I get that the consumption is high here, I mean the building is huge so it must cost a lot to run, but l'd feel more comfortable with this if I knew how much was used for heating, lighting, and other stuff. I can work that out in my home from my bill but I've got no idea here.

People were also sensitive to how the apportionment might affect the way they are viewed in comparison to others, particularly when a partial representation of carbon consumption was exposed.

Fred: l've got so many friends who think because they recycle paper at home they're being green but then they drive 50 miles a day and they're probably in the top $5 \%$ of consumers in the world of energy.

On apportionment, we conclude that the way in which energy is apportioned to consumers, particularly when measured from a communal space is key in attempting to make it accountable. Our simplistic method of apportionment was sufficient for participants to understand their effect on widespread energy monitoring and afforded them sufficient reasoning to be able to make choices about how and where they work.

\subsection{Representation}

Our third key research question concerns the adequacy and appropriateness of representations of apportionment across and between multiple spaces - do they suffice to 
make the distributed character of energy consumption accountable to consumers, and in what ways? Our elaboration of the previous issues concerning the measurement and apportionment of widespread energy measurement for accountability already provide some insight into this; here we go into more detail.

Given that some of the recruits were friends and were likely to visit one another's homes, coupled with the sensitizing effect of coverage of location-based services in the media, it was anticipated that discussions surrounding data ownership and privacy would arise from use of the application. While not frequent, there were cases during the study where users visited one another, and in the interviews participants spoke about whom they would like to share data with and whom they would not:

Cecile: I really don't like the idea of people I don't know being able to see my energy consumption. It's fair if l've invited someone to my house for them to see and share part of my energy consumption but I don't want random robbers seeing my consumption.

The issue here seemed to focus on control of disclosure and an associated view that friends had access to energy consumption as part of visiting the home.

Reflecting general concerns about the security of the Internet, participants raised issues around the sensitive nature of the data collected by the system. The concerns related to what might happen if people were to access the collected energy data and use it for ill gain. Sharing of location was viewed as less concerning than the sharing of data from the home. For the majority of participants in the study, there was an acceptance of location sharing as being part of modern every day life, with understanding around mobile applications that make use of locational data.

Alice: I can tell you what I share my location with at the moment, [points to apps on phone]

Movies, Facebook, Latitude, Foursquare, Voucher Cloud.

To this we would add that the data also reflected routine patterns of consumption and activity. The dashboard enabled participants to reason about routines and how these impacted their consumption across different spaces. Unsurprisingly, a regular pattern of consumption and space occupation was common in the data. People could readily see their activities in the data and provide accounts and rationales for the routines. Consider, for example, a week's of George's time charts that he explored in the interview using the lower chart in section 3 of the dashboard (Figure 1).

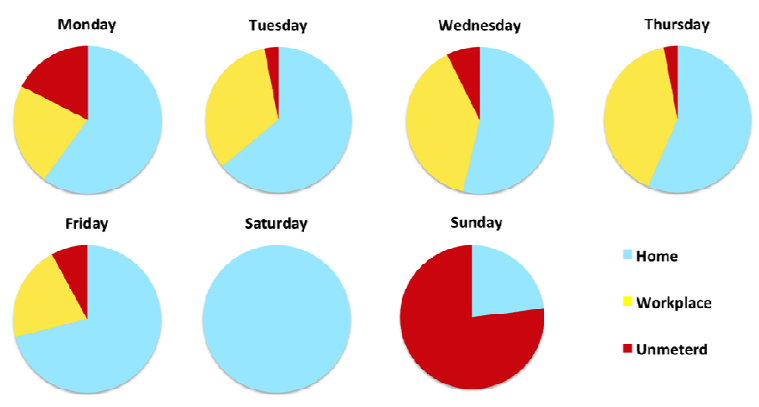

Fig. 3. A week's time apportionment charts for George 
In this week George was at work each weekday (yellow), he also had some time in unmonitored space (red). On two days the time in unmonitored space increased as he was engaged in social activities. This was revealed during his interview:

George: Ah, I left work early on that day, Monday. I was driving the wife to her piano lesson, the traffic was crap, was on the road for ages. I can see that 'cause that bit's bigger [points to unmonitored space section of chart].

On Saturday, George spent no time in communal or unmonitored space as a result of him staying at home to attend to household chores. George also paid particular attention to section 2 of the dashboard for weekend days. He noted that for the Saturday he spent at home the amount of energy consumed was higher than normal and he was able to attribute his actions to this high consumption

George: $\mathrm{Ha}$, that'll be me doing all the washing and drying then. Plenty of work done there.

The representations used made it possible for participants to identify their routines and associated consumption across multiple spaces they could easily spot significant changes to those routines and how this altered their consumption profiles.

When making the workplace consumption visible to participants through the dashboard, the accountability of that consumption to them as workers within the space was drawn into question.

Bill: I can't be held accountable for the consumption here, even if it is high 'cause I'm only the doer of the work not the one ordering it to be done, or the one who controls how these buildings are run. I mean if it were up to me l'd just work at home and then none of it could be blamed on me

Here, Bill demonstrates how he feels that he should not be held to account for consumption in the workplace, as it is someone else's job to manage it.

The dashboard thus provided an interesting means of showing the impact of their everyday activities on their daily routines and the possibilities offered by widespread energy monitoring. Our results show that presenting consumption information for different buildings occupied by a user over a day affords strong accountability for the consumption that takes place inside these buildings, provided that some apportionment of communal consumption is applied.

\section{$6 \quad$ Key Lessons and Future Challenges}

As access to energy data grows, new opportunities for systems that surface consumption for people across multiple spaces arise. However, the reactions to our probe from individuals who were subject to the data that this kind of monitoring can provide suggest that there are a number of issues that the community must consider.

\subsection{The Nature of Real World Monitoring}

Our probe highlighted the messy nature of real world data collection. Ubiquitous computing often looks to the future outlining new services or presuming the existence 
of future capabilities. For example, the vision of a global personal energy meter described by Hay et al. [16] depicts fine grain modelling and the ability to show how much energy has been used as a result of our daily activities at the level of the appliances used and activities conducted.

Our probe sought to explore the extent to which this vision may currently be realised in the real world. Our probe does not offer fine-grained measurement. The deployed infrastructure did not exploit appliance-level monitoring in the home or fine- grained monitoring in communal spaces. Rather we sought to exploit systems in widespread use or in the process of widespread deployment. Working with existing sensing systems is a key feature of real world monitoring and is likely to be a perennial feature for any energy monitoring system given the nature of the built environment where legacy plays a critical role.

Energy consumption data is often monitored for reasons other than apportionment to individual people (e.g. to support the maintenance of buildings). This means that unambiguous attribution of measurement is likely to remain technically very demanding. Gaps and seams in data were ever-present in this data collection of the probe and we would suggest this a perennial feature of this class of system.

Rather than seeing these gaps as problematic we would suggest an approach drawing upon 'seamful design' [3] to exploit unmeasured space and limited accuracy. Letting people see the gaps in data allows them to make inferences about their activities, provoking thoughtful interactions with energy. In fact, the ease with which participants could infer or recall the activities that generated their data suggests that there may not be much need to process the data further to reveal patterns: self-reporting may instead be a useful (and enjoyable) means of a user improving the accuracy of their own data.

\subsection{The Dynamic and Social Nature of Apportionment}

The probe made use of a very simple apportionment model to elicit reactions from people to the notion of apportionment for energy, particularly in communal spaces. Building management systems in communal buildings often represent consumption on a much greater scale and with less granularity than the home. This can make it difficult for individuals to relate to data from communal spaces. However, this is not the only reason for the lack of a personal view of accountability. The complex nature of our interactions with energy throughout our lives means that the principles of apportionment are highly contextualized in practice. Consequently, negotiation regarding the cause of energy use is integral to understanding energy consumption and attributing responsibility. In the home, apportionment is negotiated amongst a small, intimate social network. In larger communal spaces this network of accountability can be very broad, including people and practices unfamiliar to the individual, dissuading the individual from taking responsibility.

Ubiquitous computing systems that seek to encourage some level of personal accountability within communal spaces should consider how to leverage the broader social networks in communal spaces. Who is responsible for consumption and how much of the consumption for a space they are responsible for will change constantly 
depending upon the members of the space and roles that they take. This is not a simple issue. We suggest that the development of this kind of system should consider the need for a human interface to the apportionment models applied, allowing all actors in a system to have influence over the way in which they are made accountable for consumption.

\subsection{Disclosing Energy Data}

Participants' responses to the probe highlight the sensitive nature of energy data and awareness that energy use could tell others a great deal about an individual's everyday activity and routine. The idea of sharing energy data with other people for various reasons was accepted by our participants: the participants appeared to have an understanding that they were often donating personal data as they used their phones, computers, and other technologies, but that they tended to receive some kind of reward for doing so (improved services, financial offers, etc.). Participants' reactions to the probe indicated that it was not the inference that someone might draw from their data that was of concern, but that there appeared to be no clear mechanisms to prevent others from accessing their data without permission.

Participants drew on a range of analogies such as inviting people into the privacy of their home to suggest suitable mechanisms that they were familiar with. This highlighted how the nature of relationship greatly affects the control a user might expect over the disclosure of their data. For example, when considering disclosing energy data to a commercial partner, participants spoke of formal contracts, laying out specific terms and returns. When considering disclosure to friends and family, participants related to practices of visiting suggesting with respect to the data from their home that if they were happy to allow someone through their front door then they were happy to share their energy data with them.

This leads us to suggest that personal energy monitoring systems should provide mechanisms for controlling disclosure of personal energy data and that these mechanisms should be modelled on existing metaphors for, or be integrated with existing practices of, sharing and disclosure. These mechanisms should allow for renegotiation as and when necessary, and explicitly involve a discussion of the value returned to the individual in disclosing the data.

\subsection{The Alignment of Incentives with Consumption}

People are currently exposed to multiple incentives to reduce energy consumption. In the workplace, the motivation is often provided in terms of carbon reduction as part of initiatives that aim to improve the public image of a company or organization and reduce running costs. In the home people are exposed to raw data in the form of meters and tariffs, as this is a space in which people directly incur cost for their consumption. This direct relationship suggests the use of tariffs and individual monetary reward as an incentive for change.

Our probe also surfaced energy consumption from different spaces that users inhabit as they go about their everyday lives. This invited cross-comparison between these 
spaces and promoted user reflection on the amount of energy used in each context. Understanding and managing the transitions between these spaces and the possibility of linking and comparing energy use across them will become a critical issue to consider as energy monitoring becomes increasingly widespread.

Offering individuals greater access to their energy consumption opens up the possibility of foregrounding the different incentives and drivers towards energy reduction. In doing so, this raises issues of the extent to which these are complementary or in conflict with each other. There is an as yet unexploited opportunity to transfer good practice through common mechanisms for surfacing energy use and unifying incentives, making people accountable for their consumption across all aspects of their lives in a consistent manner, and rewarding them for managing their throughout the day.

\section{Conclusion}

We have presented the deployment of a technology probe used to elicit user reactions to the measurement, apportionment and representation of energy consumption across multiple spaces. Our probe combined energy monitoring systems from the home and the workplace allowing us to study reactions by users towards the measurement and apportion of energy use. This has involved exploiting representations of personal energy consumption to investigate accountability of consumption measured across the home and the workspace.

A striking feature of our probe was the extent to which the measurement of energy consumption intertwines with a broad range of other social, political and economic drivers. The measurement and display of energy consumption surface these in terms of issues around apportionment and accountability. The future design of any widespread energy monitoring system will, by the very nature of the energy data collected, be embedded within a range of concerns about the nature of sustainability [7].

Our probe suggests that these issues will play out in the ways in which the technology seeks to attribute energy use and the ways in which the presentation of consumption provides information about the different contexts of energy use. Most striking for us was the ways in which energy consumption at work dominated total energy use but was not matched by the ability for people to exercise control over this use.

The probe highlights the importance of understanding and aligning with the broad social, political and economic drivers at play. Measurement and presentation of energy use is unlikely to change users' behaviour without some form of initiative to encourage and reward change. It is important that we understand the initiatives and that our measurement aligns with them rather than undermines them.

Our final observation is the way in which any ubiquitous computing system designed to measure energy embodies a sense of accountability. The challenge here is the way in which accountability and attribution of use are encoded in the system. Energy apportionment and accountability is a dynamically negotiated process. For example, the apportionment of energy use from a utility can often be a source of major discussion and debate involving consideration, compromise and trading. People need to understand the rationale for apportionment at play and may often wish to negotiate the principles by which this apportionment is carried out. 


\section{References}

1. Abrahamse, W., Steg, L., Vlek, C., Rothengatter, T.: A review of intervention studies aimed at household energy conservation. Journal of Environmental Psychology 25, 273291 (2005)

2. Brynjarsdottir, H., Håkansson, M., Pierce, J., Baumer, E., DiSalvo, C., Sengers, P.: Sustainably unpersuaded: how persuasion narrows our vision of sustainability. In: Proceedings of the SIGCHI Conference on Human Factors in Computing Systems, pp. 947-956. ACM, New York (2012)

3. Chalmers, M.: Seamful Design and Ubicomp Infrastructure. In: Proceedings of Ubicomp 2003 Workshop at the Crossroads: The Interaction of HCI and Systems Issues in Ubicomp (2003)

4. Chetty, M., Brush, A., Meyers, B.: It's Not Easy Being Green: Understanding Home Computer Power Management. In: Proceedings of the SIGCHI Conference on Human Factors in Computing Systems, pp. 1033-1042. ACM, New York (2009)

5. Chetty, M., Tran, D., Grinter, R.E.: Getting to Green: Understanding Resource Consumption in the Home. In: Proceedings of the 10th International Conference on Ubiquitous Computing, pp. 242-251. ACM, New York (2008)

6. Department for Energy and Climate Change. UK Climate Change Sustainable Development Indicator: 2010 Greenhouse Gas Emissions, Provisional Figures. Technical report, Department for Energy and Climate Change (2011)

7. Disalvo, C., Sengers, P., Brynjarsdóttir, H.: Mapping the landscape of sustainable HCI. In: Proceedings of the SIGCHI Conference on Human Factors in Computing Systems, pp. 1975-1984. ACM, New York (2010)

8. Ehrhardt-Martinez, K., Donnelly, K.A., Laitner, J.A.: Advanced metering initiatives and residential feedback programs: A meta-review for household electricity-saving opportunities. American Council for an Energy-Efficient Economy, Washington, D.C. (2010)

9. Environmental Audit Committee. Personal Carbon Trading. Fifth Report of Session 20072008. House of Commons (2008),

http: / / www.publications.parliament.uk/pa/cm200708/ cmselect/cmenvaud/565/565.pdf

10. Froehlich, J., Dillahunt, T., Klasnja, P., Mankoff, S.C., Harrison, B., Landay, J.A.: UbiGreen: Investigating a Mobile Tool for Tracking and Supporting Green Transportation Habits. In: Proceedings of the SIGCHI Conference on Human Factors in Computing Systems, pp. 1043-1052. ACM, New York (2009)

11. Froehlich, J., Findlater, L., Landay, J.: The design of eco-feedback technology. In: Proceedings of the SIGCHI Conference on Human Factors in Computing Systems, pp. 19992008. ACM, New York (2010)

12. Gaver, W., Dunne, A.: Projected Realities Conceptual Design for Cultural Effect. In: Proceedings of the SIGCHI Conference on Human Factors in Computing Systems, pp. 600607. ACM, New York (1999)

13. Gustafsson, A., Gyllenswärd, M.: The Power-Aware Cord: Energy Awareness through Ambient Information Display. In: CHI 2005 Extended Abstracts on Human Factors in Computing Systems, pp. 1423-1426. ACM, New York (2005)

14. Gyllensward, M., Gustafsson, A., Bang, M.: Visualizing Energy Consumption of Radiators. In: IJsselsteijn, W.A., de Kort, Y.A.W., Midden, C., Eggen, B., van den Hoven, E. (eds.) PERSUASIVE 2006. LNCS, vol. 3962, pp. 167-170. Springer, Heidelberg (2006) 
15. Hay, S., Rice, A.: The case for apportionment. In: Proceedings of the First ACM Workshop on Embedded Sensing Systems for Energy-Efficiency in Buildings, pp. 13-18. ACM, New York (2009)

16. Hay, S., Rice, A.: A global personal energy meter. Adjunct Proceedings of the 7th International Conference on Pervasive Computing (2009)

17. Heller, F., Borchers, J.: PowerSocket: towards on-outlet power consumption visualization. In: CHI 2011 Extended Abstracts on Human Factors in Computing Systems, pp. 19811986. ACM, New York (2011)

18. Hutchinson, H., Hansen, H., Roussel, N., Eiderbäck, B., Mackay, W., Westerlund, B., Bederson, B.B., Druin, A., Plaisant, C., Beaudouin-Lafon, M., Conversy, S., Evans, H.: Technology Probes: Inspiring Design for and with Families. In: Proceedings of the SIGCHI Conference on Human Factors in Computing Systems, pp. 17-24. ACM, New York (2003)

19. Jönsson, L., Broms, L., Katzeff, C.: Watt-Lite: energy statistics made tangible. In: Proceedings of the 8th ACM Conference on Designing Interactive Systems, pp. 240-243. ACM, New York (2010)

20. Mun, M., Reddy, S., Shilton, K., Yau, N., Burke, J., Estrin, D., Hansen, M., Howard, E., West, R., Boda, P.: PEIR, the personal environmental impact report, as a platform for participatory sensing systems research. In: Proceedings of the 7th International Conference on Mobile Systems, Applications, and Services, pp. 55-68. ACM, New York (2009)

21. Patel, S.N., Robertson, T., Kientz, J.A., Reynolds, M.S., Abowd, G.D.: At the flick of a switch: Detecting and classifying unique electrical events on the residential power line (Nominated for the best paper award). In: Krumm, J., Abowd, G.D., Seneviratne, A., Strang, T. (eds.) UbiComp 2007. LNCS, vol. 4717, pp. 271-288. Springer, Heidelberg (2007)

22. Pierce, J., Paulos, E.: Beyond energy monitors: interaction, energy, and emerging energy systems. In: Proceedings of the SIGCHI Conference on Human Factors in Computing Systems, pp. 665-674. ACM, New York (2012)

23. Schwartz, T., Betz, M., Ramirez, L.: Sustainable energy practices at work. In: Proceedings of the 6th Nordic Conference on Human-Computer Interaction: Extending Boundaries, pp. 452-462. ACM, New York (2010)

24. Yun, T.-J.: Investigating the impact of a minimalist in-home energy consumption display. In: CHI 2009 Extended Abstracts on Human Factors in Computing Systems, pp. 44174422. ACM, New York (2009) 